\title{
A Data-Driven Soft Sensor to Forecast Energy Consumption in Wastewater Treatment Plants: A Case Study
}

Fouzi Harrou, Member, IEEE, Tuoyuan Cheng, Ying Sun, TorOve Leiknes, Noreddine Ghaffour

\begin{abstract}
Energy consumption is vital to the global costs of wastewater treatment plants (WWTPs). With the increase of installed WWTPs worldwide, the modeling and forecast of their energy consumption have become a critical factor in WWTP design to meet environmental and economic requirements. The accurate and swift energy consumption forecasting soft-sensors are not only supportive to the daily electric and financial budgeting by WWTP practitioners on the micro-scale, but also beneficial to local municipal operation and fundamental to regional environmental impact estimation on the macro-scale. Energy consumption in WWTPs is influenced by different biological and environmental factors, making it complicated and challenging to build soft-sensors. This paper intends to provide short-term forecasting of WWTP energy consumption based on data-driven soft sensors using traditional time-series and deep learning methods. Ten data-driven soft sensors, including the ordinary least square, exponential smoothing state space, local regression, auto-regressive integrated moving average (ARIMA), structural time series model, Bayesian structural time series, non-linear auto-regressive, long short-term memory with and without updates, and gated recurrent units have been investigated and compared for WWTP energy consumption forecasting. Energy consumption time-series data from a membrane bioreactor-based WWTP in the middle east is used to evaluate the performances of the proposed soft-sensors. Results showed that ARIMA achieved slightly improved performances, among others. The employment of adaptive deep learning-based soft sensors is expected to enhance the capabilities of the deep models to quickly and accurately follow the trend of future data.
\end{abstract}

Index Terms - WWTP energy consumption, data-driven soft sensor, deep learning, time-series forecasting

\section{NOMENCLATURE}

ACF: Auto-correlation function

AR: Auto-regressive

ARIMA: Auto-regressive integrated moving average

BSTS: Bayesian structural time series

ES: $\quad$ Exponential smoothing state space

LSTM and LSTM_wUpdates: Long short-term memory and Long short-term memory with updates

LSTM_woUpdates: LSTM without updates

MA: Moving average

MAE: Mean absolute error

MAPE: Mean absolute percentage error

MBR: Membrane bioreactor

NAR: Non-linear auto-regressive

OLS: Ordinary least square

RMSE: Root mean square error

RMSLE: Root mean squared log error

F. Harrou, and Y. Sun are with Computer, Electrical and Mathematical Sciences and Engineering (CEMSE) Division, KAUST, Thuwal, 23955-6900, Saudi Arabia, Email: fouzi.harrou@kaust.edu.sa

T. Cheng, T. Leiknes, and N. Ghaffour are with Water Desalination and Reuse Center, Biological and Environmental Science and Engineering (BESE) Division, King Abdullah University of Science and Technology (KAUST), Thuwal, 23955-6900, Saudi Arabia, Email: tuoyuan.cheng@kaust.edu.sa

This work was supported by funding from King Abdullah University of Science and Technology (KAUST), Office of Sponsored Research (OSR) under Award No: OSR-2019-CRG7-3800.
SDL: Seasonal decomposition by local regression

STS: Structural time series

WWTP: Wastewater treatment plant

GRU and GRU_wUpdates: Gated recurrent unit and Gated recurrent unit with updates

\section{INTRODUCTION}

$\mathrm{T}$ HE modern water sector is receiving its fourth revolution, which demands innovative water conservation technologies and closed water loops [1]. One promising solution is from the wastewater treatment plant (WWTP), where wastewater is not only minimizing aqueous pollution by maximizing reclaimed water and nutrients [2] but also offering voluminous data [3]. Concurrently, the introduction of artificial intelligence brings a digital transition to the water sector, which will change the role and performance of infrastructure services [4], [5].

WWTPs are designed to remove pollutants, to reach predefined effluent quality with minimum expenditure. To monitor the result and efficiency during the wastewater treatment processes, WWTP operators employ sensors to record multiple parameters regularly [6], [7]. As one of the most significant indicators, the power consumption of the plant is directly linked to greenhouse gas emission and economic input of the regional environmental protection [8]. Therefore, practitioners attach decisive importance to the analysis and forecast soft-sensors of plant-wise power consumption time series. The accurate and swift 
energy consumption forecasting soft-sensors are not only supportive to the daily electric and economic budgeting by WWTP practitioners on the micro-scale, but also beneficial to local municipal operation and fundamental to regional environmental impact estimation on the macroscale.

Membrane bioreactor (MBR) is a promising solution to the water scarcity in water-stressed regions such as the middle east. MBR effluent is of hygiene quality, which allows for irrigation or potable reuse [9]. Involving pretreatment, aeration, pumping, and membrane filtration, WWTPs with MBR installed are energy-intensive, which asks for adequate energy consumption monitoring as well as accurate forecasting soft-sensors [10]. However, until recently, limited information is reported on energy consumption soft-sensors for plant-scale MBR in the middle east.

The modeling of energy consumption soft-sensors is challenged by the complex composition of incoming wastewater and the convoluted physical, biological, physio-chemical mechanisms of treatment systems, as well as the non-linear, dynamic, and periodic nature of environmental systems. It is reported in the literature that around $25-40 \%$ of operating costs are defrayed for energy consumption [11]-[13]. Several studies have been focused on analyzing wastewater energy consumption and efficiency [12]. For instance, in [13], a multi-step approach has been introduced to evaluate WWTP energy efficiency in Italy, together with suggestions on improving energy efficiency. In [14], a method based on non-radial data envelopment analysis is proposed to compute energy efficiency metrics to several WWTPs in Spain. In [15], a gray model is introduced to forecast WWTP discharge and energy consumption in China.

To obtain more accurate information about the operational evolution of complex processes, additional hardware sensors can be installed to the process components. However, this will lead to additional costs, extra space for sensors installments, as well as calibration and maintenance of installed sensors. As an alternative, soft sensors (or virtual sensors) use analytical redundancy underlying relations between the measured process observations to estimate unmeasured or expensive-to-measure process variables. Nowadays, data-driven soft sensorsbased methods are becoming more popular [16]. Among existing soft sensors, traditional soft sensors based on time-series models are the most widely used ones [14]. Parametric models, such as the auto-regressive model and its variants can achieve a reasonable performance when time-series data shows regular variations, but they may provide unsatisfactory forecast accuracy when the time series have irregular variations. To bypass this limitation, researchers developed non-parametric models. In [17], radial basis function neural networks and multi-variable linear regression soft-sensors are applied to forecast energy consumption in WWTP. In addition, fuzzy clustering algorithm is applied to categorize the data and analyze the correlation between energy consumption and impact factors. In [18], neural networks and random forest were combined to model energy cost in WWTP. It has been shown that these flexible machine learning soft-sensors may outperform the traditional linear regression models. Aside from theory-based science approaches (i.e., applying mechanistic models or empirical knowledge), nowadays the modeling methodologies using theory-free data-driven soft-sensors are gaining more attention [19].

The above described shallow soft sensors are not specifically targeting implicit and relevant information embedded in time-series data. Recently, soft-sensor design via deep learning has emerged as a promising line of research in modeling and forecasting time series data, both in the academia and the industry [20]. In fact, soft sensor-based deep learning models are powerful tools to model implicit relationships between process variables, to enable complicated pattern recognition, and to describe time dependencies in time series data. For instance, in [21], a flexible soft sensor method based on deep learning is introduced for CO content estimation. This soft sensor merges the desirable characteristics of deep learning of semi-supervised process data with a hierarchical extreme learning machine (HELM). Specifically, the basic HELM is firstly transformed into a semisupervised approach using the manifold regularization procedure. The autoencoder deep learning model is applied as a feature extractor to the labeled and unlabeled data. Then, the semisupervised ELM is employed as a regressor at the last hidden layer. The performance of this approach has been verified to estimate the carbon monoxide content in a high-low transformer and showed superior performance compared to conventional methods. In [20], a soft sensor monitoring approach merging deep learning models with a one-class support vector machine (SVM) is proposed to detect influent measurements that affect treatment units states of WWTP. The soft sensor approach uses recurrent neural networks (RNNs)-based model to learn time-dependent and extract relevant features from multivariate time series, and applied a one-class SVM algorithm to detect abnormal events in influent measurements (e.g. seawater intrusion, water supply shut-down, discharge from construction area, and lift station maintenance/flushing). The method in [22] considers a data-driven soft sensor approach based on neural networks for sensor validation. To this end, a multilayer perceptron (MLP) estimator is applied as a virtual sensor for estimating and replacing measurements of unreliable sensors, and MLP with a softmax output is employed for detecting and isolating faults. Numerical results based on air quality chemical multi-sensor data showed the suitable performance of this soft sensor approach. In deep learning soft sensor methods, Long short-term memory (LSTM) neural networks and gated recurrent unit (GRU) neural networks are regarded as effective in modeling timedependent data with longer time spans [23].

In this paper, data-driven soft sensors to forecast WWTP energy consumption using advanced structures 
of deep learning methods and conventional forecasting methods are presented.

- We would also investigate the performance of deep learning-based soft-sensors on datasets of limited size.

- Furthermore, we investigate whether deep learningbased soft sensors can outperform conventional soft sensors with relatively small-sized energy consumption data. This study also aims to build and compare data-driven soft sensors to forecast the electric power consumption time series of an MBR-based WWTP in the middle east. At first, LSTM and GRU models are adopted as data-driven soft-sensors to forecast WWTP energy conversion. To the best of our knowledge, this is the first time that the LSTM and GRU are involved in improving forecasts of WWTP energy consumption time-series data. To eventually improve the forecasting performance of LSTM and GRU, their adaptive versions are investigated.

- Besides, this study conducts a performance comparison with several soft sensors based on traditional statistical methods, including ordinary least square (OLS), seasonal decomposition by local regression (SDL), exponential smoothing state space (ES), auto-regressive integrated moving average (ARIMA), structural time series (STS), Bayesian structural time series (BSTS), and non-linear autoregressive (NAR) artificial neural networks. Aside from traditional methods, deep learning models are fitted then assessed by several metrics. The OLS as a classical regression model is built based on a window of previous 30 days records. The ES, as a classical signal processing model, is constructed to assign exponentially decreasing weights over time. The seasonal decomposition of time series by SDL as a model involving the trend, seasonality, and error decomposition is built to consider long-term and periodic components in a univariate time series. The ARIMA is used as a classical time series model. The STS model uses local trends and additional seasonal components, while the BSTS uses a sparse AR process, semi-local linear trend, and seasonal components. The NAR is applied as a classical shallow neural network model, while LSTM and GRU are involved as recent deep neural network models [24]. Energy consumption time-series data from an MBR-based WWTP located in Saudi Arabia is adopted to evaluate the performance of data-driven forecasting models.

The MBR-based WWTP power consumption dataset and the modeling specifics were presented in section II. The results and discussions were given in section IV to show model performances and comparisons. The conclusions were drawn in section $\mathrm{V}$.

\section{MATERIALS AND METHODS}

\section{A. Wastewater treatment plant (WWTP) description}

The electric power consumption time series of a municipal MBR-based WWTP in Saudi Arabia is investigated for this study [6], [16], [20]. With membrane filtration and chlorination processes installed after biological treatments, the effluent from this plant is reused for irrigation. The membrane modules are shown in Fig.1. A private company operating the plant introduced the dataset for this study. The operators receive the municipal wastewater from residents and treat the inflow via several physical, chemical, and biological processes until it reaches a predefined effluent standard and is thus dischargeable. During the whole process, pumping, aeration, and membrane filtration contribute to most electric power consumption. Suitable forecast soft-sensors would directly support managers in budgeting and operating, which asks for this research. The electric power consumption is recorded and reported daily. Here, we used daily power consumption records time-series of seven years (from 2010 to 2017). Power consumption is the overall summary of energy input or greenhouse gas emissions to meet the required aqueous environmental effluent discharge standards given initial influent conditions. Among the 2558 daily records, the plant, on average, discharges $4419 \mathrm{~m}^{3}$ treated effluents per day and uses $2.51 \mathrm{kWh} / \mathrm{m}^{3}$ in unit energy consumption. The overall power consumption fluctuates between $1727 \mathrm{kWh} / d$ and $21850 \mathrm{kWh} / \mathrm{d}$. This wide range shows the necessity for more refined control and demands better forecasting models. The WWTP power consumption time series are employed to fit, test, and assess proposed forecasting soft-sensors candidates.

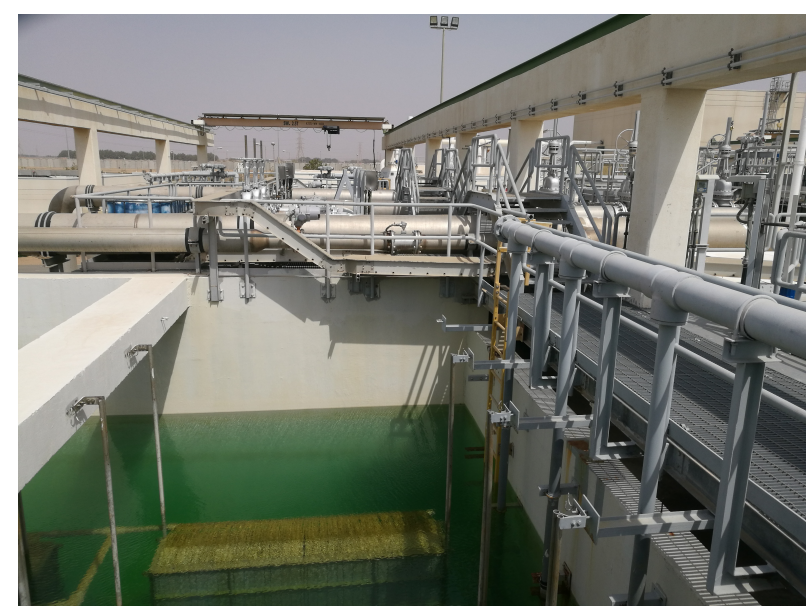

Fig. 1. The micro-filtration membrane module of the wastewater treatment plant. Filtered effluents are collected and reused for irrigation.

\section{B. Traditional statistical methods}

The ordinary least squares, as a multiple linear regression showing short-term trend line, uses the sum of squared error as its loss function. OLS assumes limited collinearity and Normal distribution of the residuals [25].

The exponential smoothing as a state-space model gives more weightings to the recent observations. ES needs to be updated frequently or otherwise, may not capture the trends well. 
The auto-regressive integrated moving average is a wellaccepted model for univariate time series forecasting [26]. As a combination of moving average and auto-regression, the best ARIMA soft-sensor is estimated per each modeling step using maximum likelihood, with the maximum order equal to 5 , and the maximum differences equal to 2. The ARIMA coefficients might be highly unstable due to variations in observations and oscillation in soft-sensors specification.

The structural time series model uses local trends and additional seasonal components, while the Bayesian STS uses a sparse AR process, semi-local linear trend, and seasonal components [27]. The STS and BSTS are relatively more interpretable by performing decomposition of the series to trends and seasonalities [28].

The NAR neural network as a classical shallow model is fitted using the Levenberg-Marquardt method for optimization on 50 neurons [29].

The OLS is fitted based on a rolling training window covering the previous 30 days records per one-step forward forecast. The ES, SDL, ARIMA, STS, BSTS, and NAR are fitted based on expanding training windows covering all previous records per one-step forward forecast [30].

\section{Long short-term memory neural networks}

Over the last decades, recurrent neural networks have been applied exponentially in machine learning. They are considered to be one of the most efficient tools for solving time-dependent forecasting tasks. Among RNN models, it is worth mentioning the long short term memory, which is the most popular and suitable for time series data forecasting [31]. As primarily designed by Hochreiter and Schmidhuber, LSTM is still actively developed for a wide range of applications [32], [33]. LSTM possesses a great capacity in describing long-term dependencies and can cope with the two common problems challenging traditional RNNs, namely the exploding and vanishing gradient problems. RNN is not suited to consider long-term time series due to the challenge of long-term dependencies. Essentially, the gradient becomes too small or large in situations with an important time step and could lead to a vanishing gradient problem. Usually, this problem occurs in the training stage, where the optimizer back-propagates and makes the process running, while the weights almost do not vary at all. To bypass this difficulty, the LSTM model, a sophisticated gated memory unit, was naturally adopted as an improved method to alleviate the vanishing gradient problems [31]. The clever concept of introducing self-loops to create paths where the gradient can flow for long durations is a central feature of the initial LSTM model [31]. LSTM is an important deep learning model that has attracted attention in time series modeling and forecasting due to its powerful ability to uncover the features of time series data within a longer time span. The LSTM schematic presentation is illustrated in Figure 2. LSTM consists of three gates regulating the information flow called input, forget, and output gates [34]. The three gates are created merely with logistic functions of weighted sums where the weights are computed by back-propagation in the training stage. The input and forget gates control the cell state.

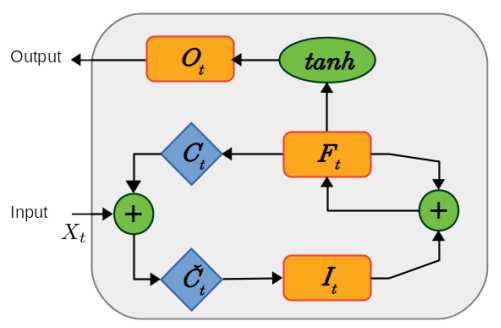

Fig. 2. Schematic illustration of LSTM unit: the three gates are represented by $\boldsymbol{I}_{\boldsymbol{t}}, \boldsymbol{F}_{\boldsymbol{t}}$, and $\boldsymbol{O}_{\boldsymbol{t}}$, the candidate memory cells and memory cell content are represented respectively by $\boldsymbol{C}$ and $\tilde{\boldsymbol{C}}$.

Let us denote the input time series as $X_{t}$, the number of hidden units as $h$, the mathematical relationship between inputs and outputs can be formulated as follows:

$$
\left\{\begin{aligned}
I_{t} & =\sigma\left(X_{t} W_{x i}+H_{t-1} W_{h i}+b_{i}\right) \\
F_{t} & =\sigma\left(X_{t} W_{x f}+H_{t-1} W_{h} f+b_{f}\right) \\
O_{t} & =\sigma\left(X_{t} W_{x o}+H_{t-1} W_{h o}+b_{o}\right) \\
\tilde{C}_{t} & =\tanh \left(X_{t} W_{x c}+H_{t-1} W_{h c}+b_{c}\right), \\
C_{t} & =F_{t} \circ C_{t-1} \circ \tilde{C}_{t} \\
H_{t} & =O_{t} \circ \tanh \left(C_{t}\right)
\end{aligned}\right.
$$

where $W_{x i}, W_{x f}, W_{x o} W_{x c}, W_{h c}$, and $W_{h i}, W_{h f}, W_{h o}$ are the weight parameters and $b_{i}, b_{f}, b_{c}$, and $b_{o}$ are bias parameters, $\circ$ denotes the element-wise multiplication. Estimating $C_{t}$ can be done by using information from memory cells $C_{t-1}$ and $\tilde{C}_{t}$.

\section{Gated recurrent unit neural networks}

Despite the suitable LSTM performance compared to other algorithms, its training needs more computation than other algorithms [35]. The expensive computation cost in LSTM training is still a challenge. GRU is essentially a special variant of LSTM introduced to optimize LSTM performance by reducing the number of LSTM parameters [35]. The significant demarcation of GRU from LSTM is that only one unit is used to control both the forgetting factor and the decision to update the unit state. GRU contains only two gates, the update gate and the reset gate, rather than previously three gates in the LSTM model (Figure 3). It is worth pointing out that there are two input variables at each time point, namely the input vector $x_{t}$ and the previous output vector $h_{t-1}$. The GRU model is come up with the concept of an update gate that provides a new evaluation procedure to calculate hidden states in RNN-based models. As in the case of LSTM models, the relationships between the reset and update gates, and other candidate hidden states are formulated as follows: 


$$
\left\{\begin{array}{l}
R_{t}=\sigma\left(X_{t} W_{x r}+H_{t-1} W_{h r}+b_{r}\right) \\
Z_{t}=\sigma\left(X_{t} W_{x z}+H_{t-1} W_{h z}+b_{z}\right) \\
\widetilde{H}_{t}=\tanh \left(X_{t} W_{x h}+\left(R_{t} \circ H_{t-1}\right) W_{h h}+b_{h}\right) \\
H_{t}=Z_{t} \circ H_{t-1}+\left(1-Z_{t}\right) \circ \widetilde{H}_{t}
\end{array}\right.
$$

where $W_{x r}, W_{x z}, W_{h r}, W_{x h}$, and $W_{h h}$ denotes weight parameters and $b_{r}, b_{z}$, and $b_{h}$ refer to bias parameters. At each time point $t$, the actual update gate $Z_{t}$ is utilized for combining the past hidden state $H_{t-1}$ and actual candidate hidden state $\tilde{H}_{t}$.

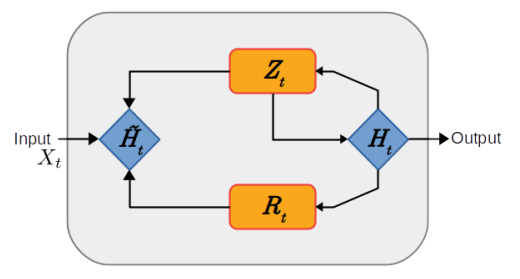

Fig. 3. GRU structure: $\boldsymbol{R}_{\boldsymbol{t}}$ and $\boldsymbol{Z}_{\boldsymbol{t}}$ denotes respectively the reset and update gates; $\boldsymbol{H}_{\boldsymbol{t}}$ and $\tilde{\boldsymbol{H}}_{\boldsymbol{t}}$ refer respectively to the candidate hidden state and hidden state.

Overall, the GRU-based soft-sensors is attractive for its shorter training time and its less parameters as compared with LSTM.

\section{DATA-BASED ENERGY CONSUMPTION FORECASTING}

Modeling and forecasting of energy consumption time series of an MBR-based WWTP is a challenging task due to the high level variability of the composition of incoming wastewater and non-linearity of environmental data. Hence, for mitigating the influence of uncertainty on the accuracy of energy consumption forecasting, this work presents a deep-learning framework to forecast energy consumption time-series of WWTP. In this paper, deep learning-based soft-sensors have been investigated and compared to power consumption forecasting. The general framework of the forecasting proposed strategies is illustrated in Figure 4. As shown in Figure 4, energy consumption is accomplished in two main stages: training and testing. The original energy consumption time-series data is split into a training sub-data and a testing sub-data. First, the raw training data is pre-processed and normalized, and then it is used to build the deep learning-based soft-sensors. The raw dataset includes ten missing points (less than 1\%), which were imputed using the expectation-maximization with bootstrapping algorithm [36]. Specifically, We normalize the time-series data by min-max normalization within the interval $[0,1]$. After the power consumption forecasting using testing data, this normalization is reversed so that the forecasted data is comparable to the original testing time-series data. The values of models' parameters are obtained by minimizing the loss function via the Adaptive Moment Estimation (Adam) optimizer based on training energy consumption data [37]. The constructed soft-sensors are then used to energy consumption in the monitored WWTP. Essentially, the deep learning-based forecasting soft-sensors learn the temporal correlation hidden on the energy consumption data and are expected to uncover and capture the sequential features in the energy consumption time series.

\section{A. Measurements of effectiveness}

For performance comparisons, the following indexes have been adopted to quantify the forecasting of energy consumption in the WWTP: Root Mean Square Error (RMSE), mean absolute error (MAE), mean absolute percentage error (MAPE), and Root Mean Squared Log Error (RMSLE).

$$
\begin{gathered}
M A E=\frac{\sum_{t=1}^{n}\left|y_{t}-\hat{y}_{t}\right|}{n}, \\
M A P E=\frac{100}{n} \sum_{t=1}^{n}\left|\frac{y_{t}-\hat{y}_{t}}{y_{t}}\right| \%, \\
R M S E=\sqrt{\frac{1}{n} \sum_{t=1}^{n}\left(y_{t}-\hat{y}_{t}\right)^{2},} \\
R M S L E=\sqrt{\frac{1}{n} \sum_{t=1}^{n}\left(\log \left(y_{t}\right)-\log \left(\hat{y}_{t}\right)\right)^{2},}
\end{gathered}
$$

where $y_{t}$ are the actual values, $\hat{y}_{t}$ are the corresponding forecasted values, and $n$ is the number of measurements. Lower RMSE, MAE, MAPE, or RMSLE values indicates more precise forecasting quality. Moreover, we shall investigate the distribution of forecasting errors through boxplots and sample autocorrelation functions (ACFs).

\section{Results AND DISCUSSION}

In this section, we would show the dataset and present forecasting assessments and discussions. In this work, we investigate the performance of deep learning-based soft sensors on datasets of limited size.

\section{A. WWTP energy consumption data analysis}

Fig. 5 shows the annual distribution of WWTP power consumption per day in 2011. We observe that the power consumption is relatively low from December to March, but rises from April to November. The equatorial climate stimulates higher water demand regionally for showering and swimming purposes from April to November. Fig. 6 displays the yearly distribution of energy consumption from 2011 to 2016. The WWTP shows improved performances in terms of power consumption, for its annual distribution shows decreases in both average values and standard deviations in the past years.

Fig. 7 presents the boxplots of weekday/weekend energy consumption at the WWTP over the entire period of data (seven years). It is shown that the WWTP energy consumption shows larger variances on Saturday. This 


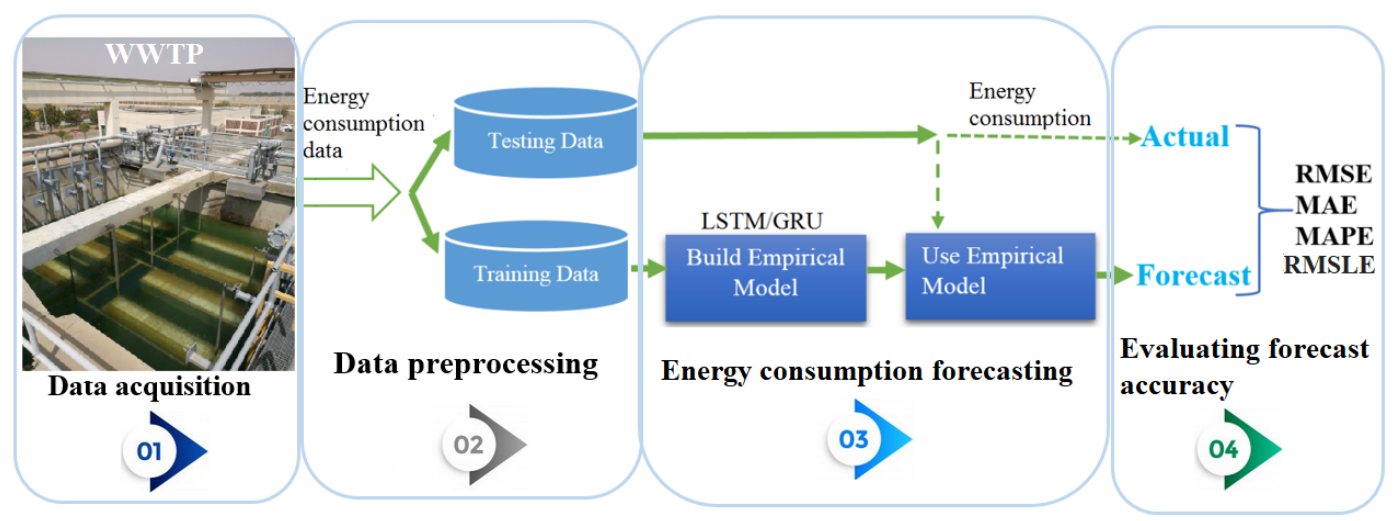

Fig. 4. Energy consumption forecasting using deep learning models.

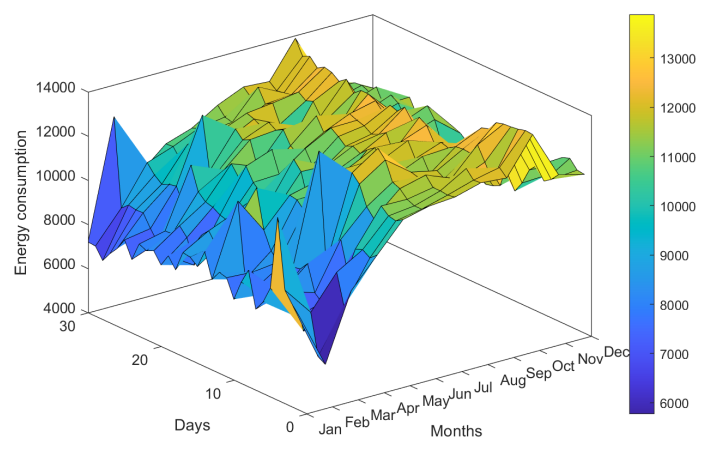

Fig. 5. Daily energy consumption recorded on 2011.

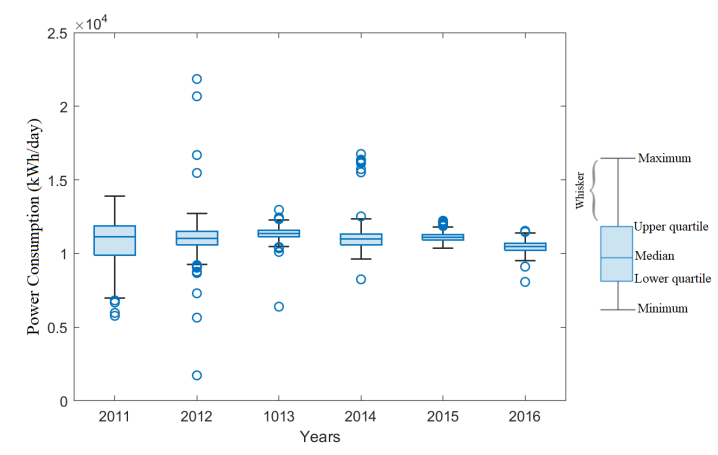

Fig. 6. Box-plots of yearly WWTP power consumption.

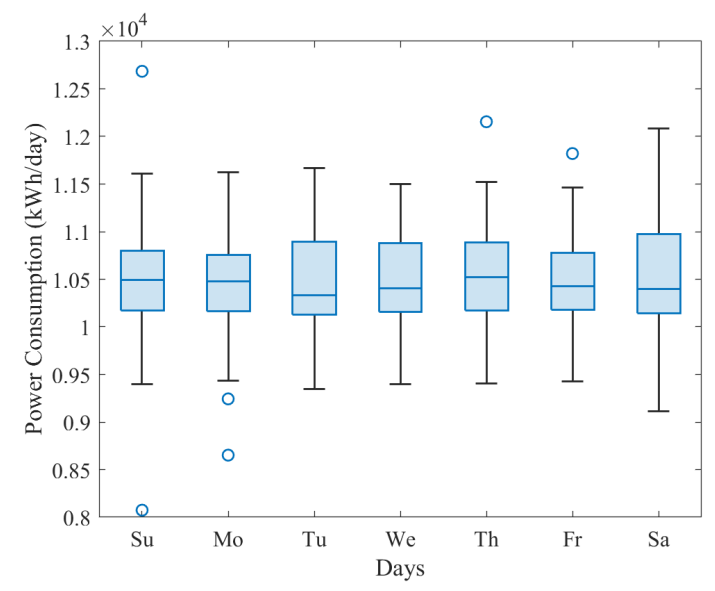

Fig. 7. WWTP energy consumption per day of all considered years. The weekend in Saudi Arabia is Friday and Saturday. is mainly due to water consumption amount affected by entertainment usages and weekly cooling tower discharges.

To assess the time dependency in the training dataset, the autocorrelation function (ACF) of energy consumption time series is illustrated in Fig. 8. ACF quantifies the correlation of a given process variable with itself at differing time lags. Fig. 8 implies periodicity in the sample $\mathrm{ACF}$ of the energy consumption time series data. There is a relatively high short-term autocorrelation (shortmemory) in this time series data. It should be noted that short-term dependence describes the low-order correlation structure of a series.

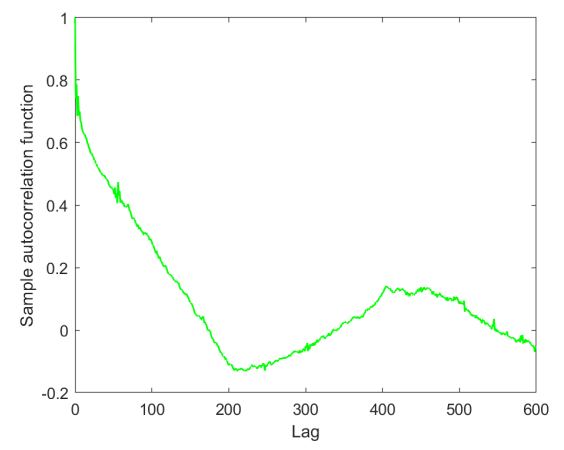

Fig. 8. Auto-correlation functions (ACFs) of the power consumption time series data.

\section{B. Forecast performance assessments}

This section will compare the forecasting performances of the above-constructed soft-sensors based on testing data. Specifically, ten models are presented and compared: OLS, ES, SDL, ARIMA, STS, BSTS, NAR, LSTM, GRU, LSTM, and GRU with updates. In LSTM-update and GRU-update, the parameters of the LSTM and GRU models are updated recursively for new arriving observations. This permits dynamically adjusting the soft-sensors to describe new testing data, and well-capturing features in the time series, which enhances the forecasting quality.

First, we train each soft-sensors based on the training energy consumption data. Then, we forecast energy consumption using the fitted models for the incoming testing 
dataset. Here, testing data consist of daily data from Sep 1st, 2016 to Sep 1st, 2017. Parameters of the constructed LSTM and GRU models obtained in the training phase are listed in Table I.

\begin{tabular}{l|c|l|c}
\hline \multicolumn{1}{c|}{ GRU parameters } & Value & \multicolumn{1}{c}{ LSTM parameters } & Value \\
\hline Droupout & 0.2 & Droupout & 0.2 \\
\hline Batch size & 64 & Batch size & \\
\hline Number of hidden units & 200 & Neural number & 200 \\
\hline Optimisation method & Adam & Optimisation method & Adam \\
\hline Loss function & MSE & Loss function & MSE \\
\hline Output activation & Relu & Output activation & Relu \\
\hline Epochs & 100 & Epochs & 100 \\
\hline Number of GRU layers & 3 & Number of LSTM layers & 1 \\
\hline Learning rate & $1.10^{-4}$ & Learning rate & 0.001 \\
\hline \multicolumn{4}{|l}{ TABLE I } \\
\multicolumn{2}{c}{ TUNED PARAMETERS IN THE GRU AND LSTM MODELS. }
\end{tabular}

As illustrations of training processes, the evolution of the RMSE as a function of the number of iterations in LSTM and GRU are displayed in Fig. 9. We can observe the convergence of the RMSE when the number of epochs is reaching 100, while the GRU-based soft-sensor converges much more efficiently than the LSTM-based soft-sensor. Therefore the GRU would require less amount of training data to fit and works well in cases when the training dataset size is small.

Fig. 9. Evolution of the RMSE value at the training stage for (a) LSTM and (b) GRU.
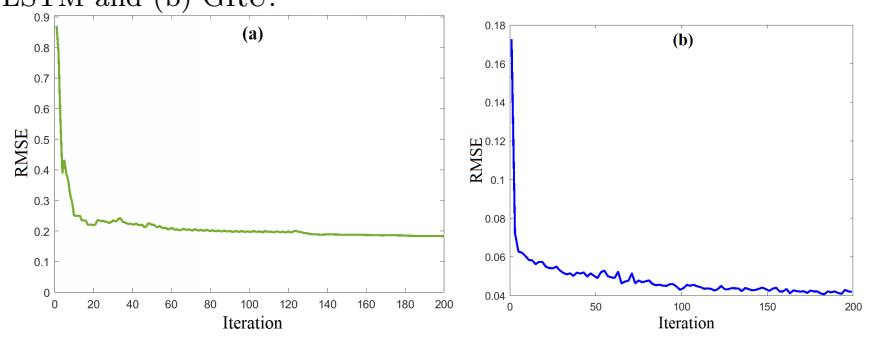

We displayed the tested together with soft-sensor forecasts time series in Fig 10. These results illustrate the advantages and disadvantages of shallow and deep learning soft-sensors for energy consumption forecasting. Also, to show clearly the accordance of the measured and forecast WWTP energy consumption from the ten models the scatter plots are presented in Fig. 11. Figure 11 shows that the forecast energy consumption from the LSTM softsensors is relatively weakly correlated with the measured WWTP energy consumption. On the other hand, the forecast energy with the other soft-sensors are strongly correlated with the measured energy consumption.

Fig. 12 shows the boxplots of the forecasting errors from each soft-sensor based on the testing data. The forecasting error is defined as the difference between the measured value $y_{t}$ and the predicted value $\hat{y}_{t}$. The method that uses shallow models (i.e., ARIMA, BSTS, ES, STS, and OLS) leads to improved forecasting of WWTP energy consumption when compared with the deep learning

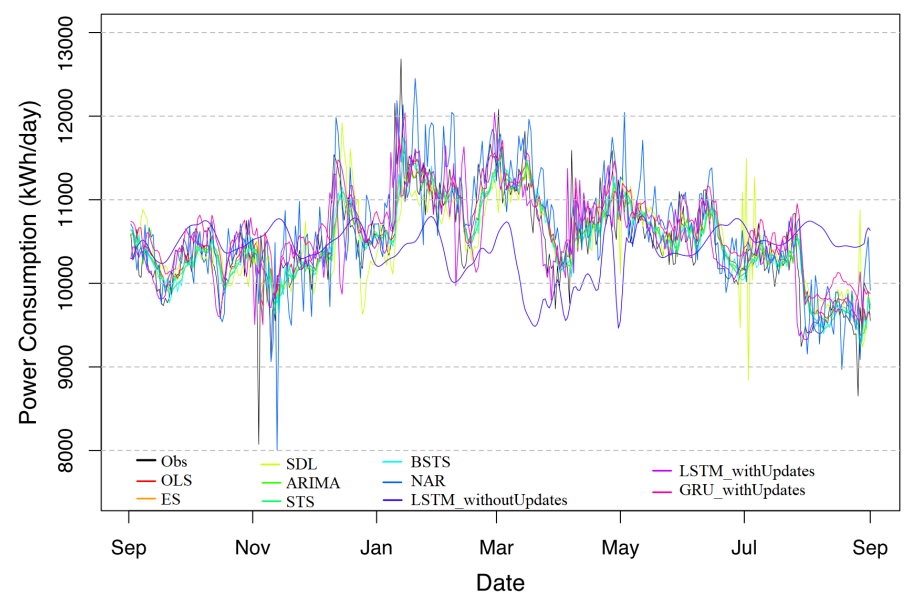

Fig. 10. Time series plot of the WWTP power consumption.

models (LSTM, and GRU). Also, it should be noted that the error analysis highlights that the forecasting accuracy obtained by the considered deep learning models can satisfy practical needs and can be useful for full-scale municipal WWTPs.

Fig. 13 shows the ACF of prediction errors obtained by each method. Theoretically, the errors as white noise process should show an autocorrelation function of zero at all lags except a value of unity at lag zero, which indicates that the process is completely uncorrelated. The ARIMA, STS, and BSTS models showed ACFs of minimal absolute values, with few lags beyond the 0.1 empirical threshold. Generally, the traditional methods produced residual ACFs of quasi-sinusoidal shapes with more values on the positive side, while the neural network methods with updates produced residual ACFs of alternative positive/negative signs or symmetric behavior around 0. This could be related to Fig. 11 that the residuals from traditional methods are of skewed distribution, while those from the neural network methods with updates are less skewed. The traditional methods are somehow systematically biased, while the deep models are less affected by distribution assumptions.

The forecasting accuracy in terms of MAE, MAPE, RMSE, and RMSLE of the ten soft-sensors when applied to test data, as well as their training time on the training data are summarized in Table II. The barplot of RMSLE values is shown in Fig. 14 to visually assist the comparison of obtained results by the ten considered soft-sensors. It can be observed that almost all models can reach MAPE ranges from $2.29 \%$ to $5.11 \%$, which means that the considered soft-sensors can follow the future trend of energy consumption. The highest forecasting accuracy was given by ARIMA by reaching MAPE of $2.29 \%$ highlighting that the forecasting error is small and the forecast and tested data are in concordance. Also, ARIMA exhibited superior performance compared to adaptive LSTM and GRU (2.29\% vs. $2.42 \%$ (LSTM) and $2.99 \%$ (GRU)). As to the training time, conventional models showed limited computational complexity as compared with deep models, 

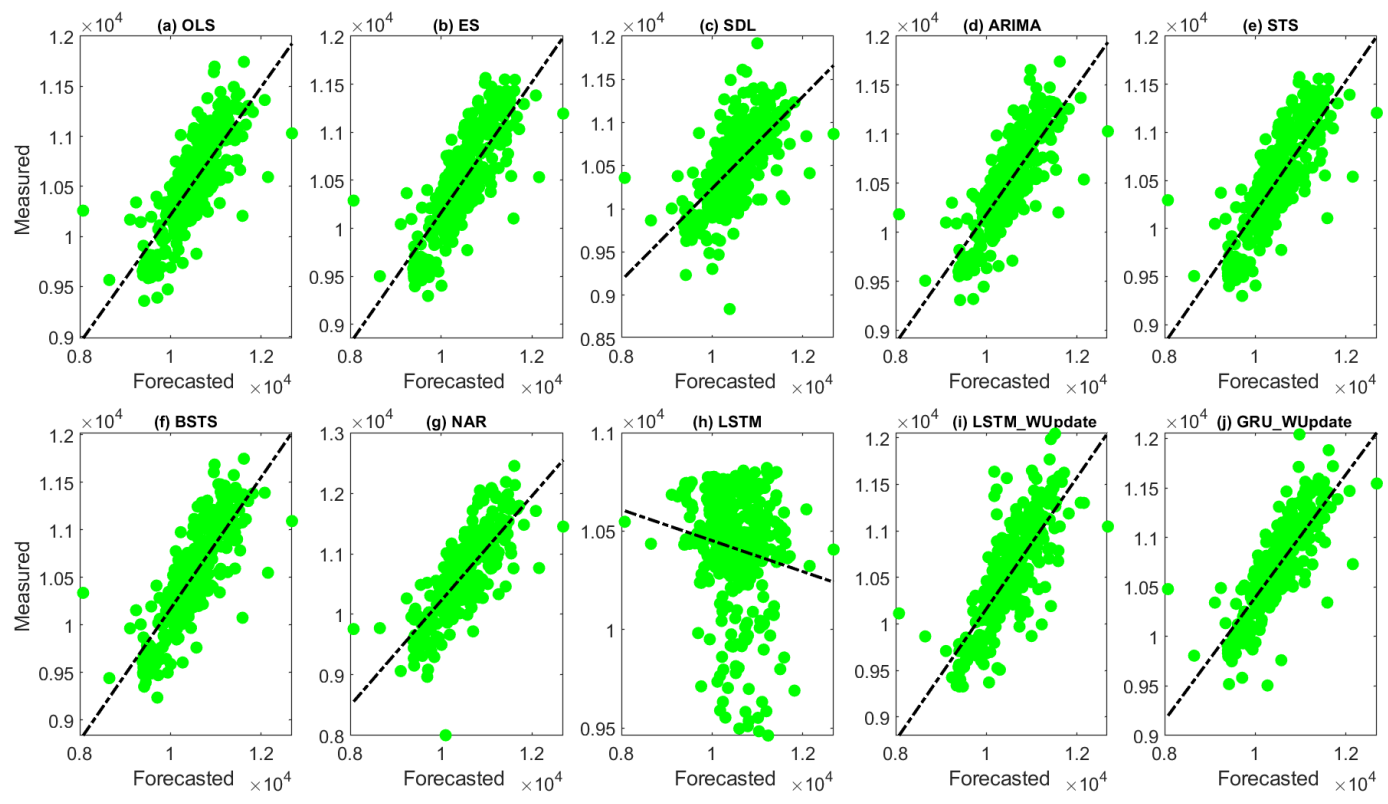

Fig. 11. Scatter plot of measured vs. forecast power consumption.

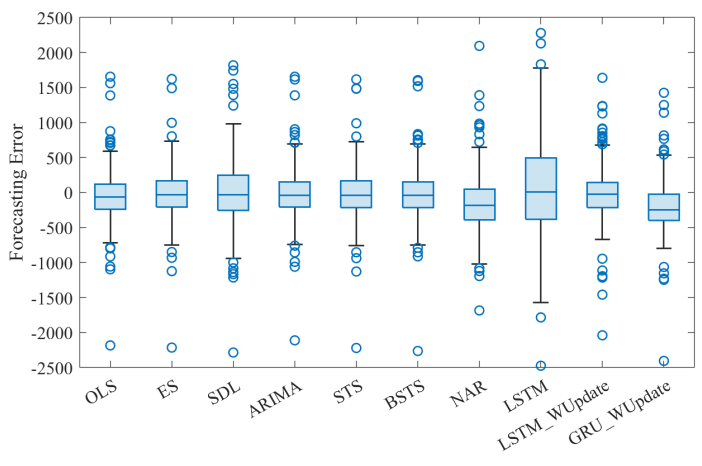

Fig. 12. Boxplots of forecasting errors from the testing dataset.

except NAR where back propagation is involved and BSTS in which iterative sampling processes were involved. All models showed acceptable training time that are much shorter than the sampling interval. Therefore, they are all usable for WWTP power consumption online forecasting. In this application, the forecasting results support that the shallow forecasting models have higher forecasting accuracy than the deep learning models (LSTM and GRU). This study highlights the improved performance of the conventional statistical soft-sensors in forecasting power consumption with a limited sample size compared to the advanced deep learning structures (i.e., LSTM and GRU). Also, deep learning models provide acceptable forecasting accuracy even with this limited dataset, due to their great capacity to efficiently learning time-dependent in times series data.

In summary, this study studied whether the performance of the advanced deep models (LSTM and GRU) is superior to conventional methods for forecasting energy consumption with a small dataset. Results indicate that deep learning models applied to small energy consump-
TABLE II

EVALUTION OF EACH FORECASTING APPROACH.

\begin{tabular}{lrrrrr}
\hline & MAE & MAPE & RMSE & RMSLE & Training Time (s) \\
\hline ARIMA & 239.38 & 2.29 & 338.20 & 3.25 & 0.53 \\
BSTS & 242.10 & 2.31 & 343.09 & 3.29 & 39.02 \\
ES & 244.66 & 2.34 & 343.23 & 3.30 & 0.17 \\
STS & 244.93 & 2.34 & 343.41 & 3.30 & 0.04 \\
OLS & 245.53 & 2.36 & 344.46 & 3.31 & 0.02 \\
LSTM_wUpdates & 253.78 & 2.42 & 371.08 & 3.54 & 377 \\
GRU_wUpdates & 307.33 & 2.99 & 396.62 & 3.84 & 321 \\
NAR & 333.78 & 3.19 & 435.99 & 4.16 & 7.72 \\
SDL & 322.70 & 3.09 & 439.55 & 4.22 & 0.18 \\
LSTM_woUpdates & 534.62 & 5.11 & 688.84 & 6.63 & 366 \\
\hline
\end{tabular}

tion datasets provide acceptable forecasting performance and could be a choice when large WWTP datasets are unavailable. The overall forecasting performance of both adaptive LSTM and GRU deep models were satisfying. The forecasting comparison results suggest that the conventional methods, namely ARIMA, BSTS, ES, STS, and OLS, slightly outperformed the deep learning methods on the tested historical records from an MBR-based WWTP from Saudi Arabia. Specifically, in this study, ARIMA achieved superior performance with a MAPE of $2.29 \%$, and the adaptive LSTM and GRU can obtain MAPE values of $2.42 \%$ and $2.99 \%$, respectively. Traditional forecasting methods, such as ARIMA, provides slightly better short-term forecasting of uni-variate energy consumption time series than deep learning models. The traditional methods focusing on the linear relationships and temporal dependencies are manually diagnosed and fixed. On the other hand, as deep learning models (LSTM and GRU) are equipped to learn long term correlations in a time series data, they could provide improved forecasting results when applied to a large amount of data.

\section{Conclusions}

Energy management of WWTPs is crucial for optimizing their operations and for energy saving. The accurate 

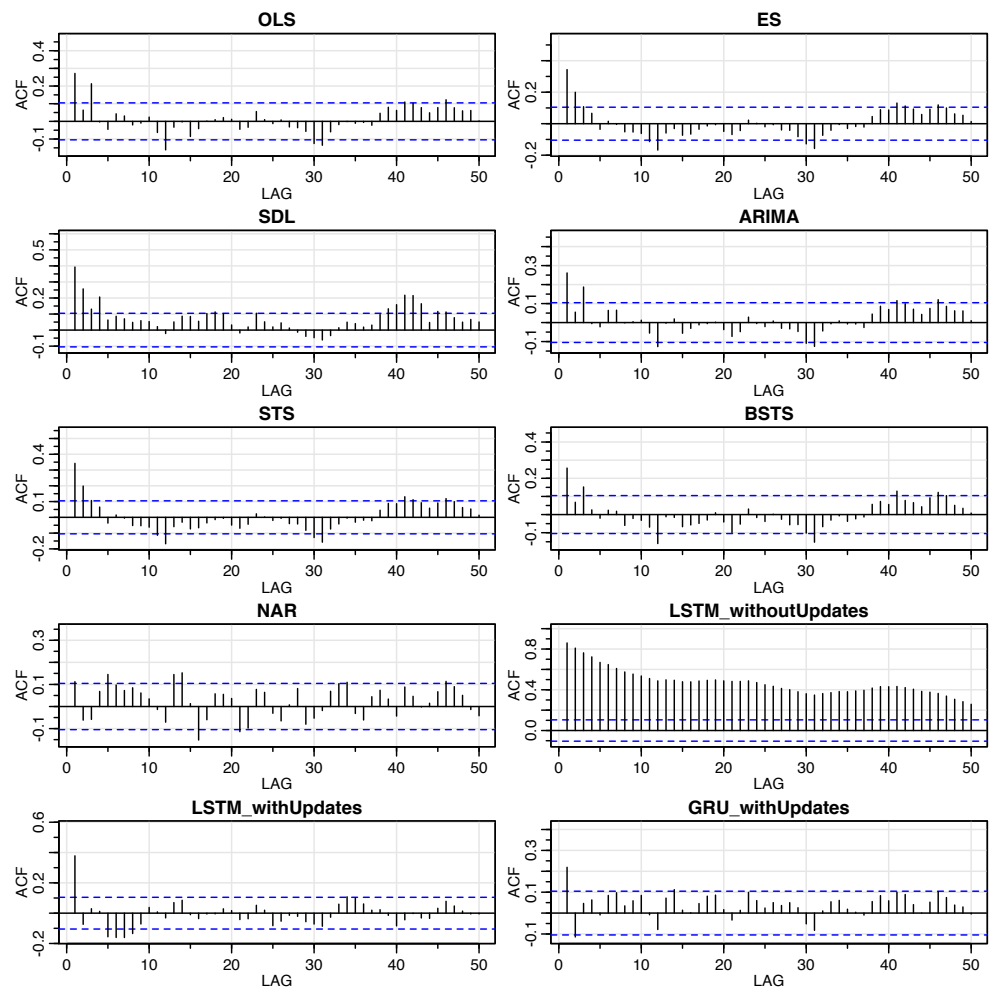

Fig. 13. ACF of forecast errors on the testing set.

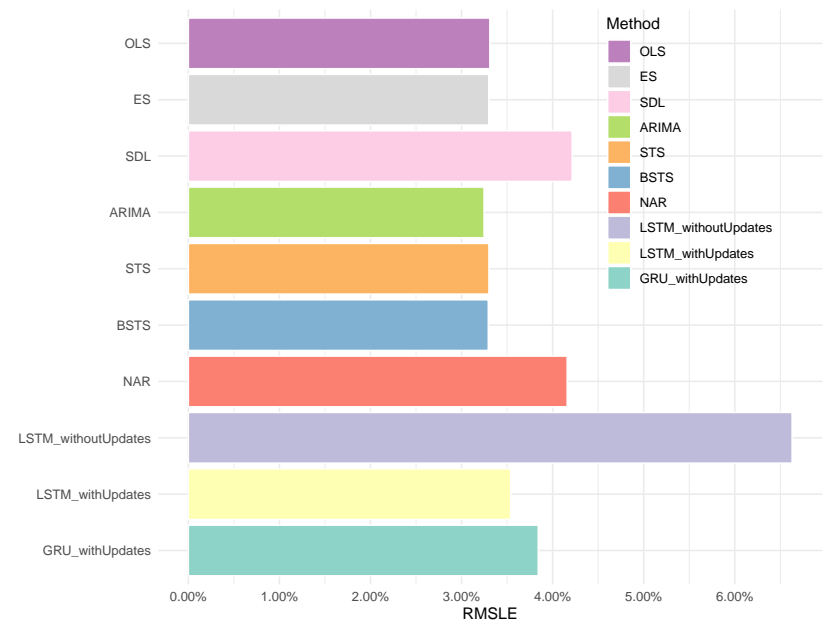

Fig. 14. Barplot of RMSLE values obtained by each forecasting approach.

and swift energy consumption forecasting models are not only supportive to the daily electric and economic budgeting by WWTP practitioners on the micro-scale, but also beneficial to local municipal operation and fundamental to regional environmental impact estimation on the macro-scale. This paper investigated and compared ten shallow and deep learning models for forecasting energy consumption is a WWTP. Data from an MBR-based WWTP located in Saudi Arabia is employed to verify the performance of the considered forecasting methods. Essentially, this study aims to provide a comparative analysis and to shed light on the performance of these advanced forecasting methods when applied to small energy consumption data sets. Comparing the proposed deep learning-based soft sensors and conventional forecasting methods based on relatively small-sized energy consumption data is investigated. The results showed that traditional methods achieved slightly improved forecasting than deep learning models in the studied small dataset.

Though built on plant-wise daily records, the training process is limited by the deficiency of data. With larger data volumes, deep learning models can offer efficient longterm forecasting. For future work, we plan to build and test soft-sensors on a dataset with expanded dimensions and higher real-time sampling frequency. As a promising candidate, multi-modal or multi-task hybrid deep learning method [38] would be investigated to build hybrid soft sensors and enhance the forecasting performance of WWTP power consumption.

\section{REFERENCES}

[1] D. Sedlak, Water 4.0: the past, present, and future of the world's most vital resource. Yale University Press, 2014.

[2] B. Wu, R. Wang, and A. G. Fane, "The roles of bacteriophages in membrane-based water and wastewater treatment processes: A review," Water research, vol. 110, pp. 120-132, 2017.

[3] L. Corominas, M. Garrido-Baserba, K. Villez, G. Olsson, U. Cortés, and M. Poch, "Transforming data into knowledge for improved wastewater treatment operation: A critical review of techniques," Environmental modelling \& software, vol. 106, pp. 89-103, 2018

[4] M. Garrido-Baserba, L. Corominas, U. Cortés, D. Rosso, and M. Poch, "The fourth-revolution in the water sector encounters the digital revolution," Environmental Science \& Technology, vol. 54 , no. 8 , pp. $4698-4705,2020$. 
[5] A. Lay-Ekuakille, G. Griffo, M. Fazio, and M. Villari, "A distributed edge computing architecture to support sensing and detecting leaks in waterworks based on advanced FDM," IEEE Sensors Journal, vol. 17, no. 23, pp. 7820-7827, 2017.

[6] T. Cheng, A. Dairi, F. Harrou, Y. Sun, and T. Leiknes, "Monitoring influent conditions of wastewater treatment plants by nonlinear data-based techniques," IEEE Access, vol. 7, pp. 108 827-108 837, 2019.

[7] J. Cleary, C. Slater, C. McGraw, and D. Diamond, "An autonomous microfluidic sensor for phosphate: On-site analysis of treated wastewater," IEEE Sensors Journal, vol. 8, no. 5, pp. $508-515,2008$

[8] Y. He, Y. Zhu, J. Chen, M. Huang, P. Wang, G. Wang, W. Zou, and G. Zhou, "Assessment of energy consumption of municipal wastewater treatment plants in china," Journal of Cleaner Production, vol. 228, pp. 399-404, 2019.

[9] L. Fortunato, M. Li, T. Cheng, Z. U. Rehman, W. Heidrich, and T. Leiknes, "Cake layer characterization in activated sludge membrane bioreactors: real-time analysis," Journal of Membrane Science, vol. 578, pp. 163-171, 2019.

[10] F. Harrou, A. Dairi, Y. Sun, and M. Senouci, "Statistical monitoring of a wastewater treatment plant: A case study," Journal of environmental management, vol. 223, pp. 807-814, 2018.

[11] G. Venkatesh and H. Brattebø, "Energy consumption, costs and environmental impacts for urban water cycle services: Case study of oslo (norway)," Energy, vol. 36, no. 2, pp. 792-800, 2011.

[12] J. Elías-Maxil, J. P. Van Der Hoek, J. Hofman, and L. Rietveld, "Energy in the urban water cycle: Actions to reduce the total expenditure of fossil fuels with emphasis on heat reclamation from urban water," Renewable and Sustainable Energy Reviews, vol. 30, pp. 808-820, 2014.

[13] D. Panepinto, S. Fiore, M. Zappone, G. Genon, and L. Meucci, "Evaluation of the energy efficiency of a large wastewater treatment plant in italy," Applied Energy, vol. 161, pp. 404-411, 2016.

[14] F. Hernández-Sancho, M. Molinos-Senante, and R. SalaGarrido, "Energy efficiency in spanish wastewater treatment plants: A non-radial dea approach," Science of the Total Environment, vol. 409, no. 14, pp. 2693-2699, 2011.

[15] Z. Li, Z. Zou, and Y. Yu, "Forecasting of wastewater discharge and the energy consumption in china based on grey model," Mathematical Problems in Engineering, vol. 2019, 2019.

[16] T. Cheng, F. Harrou, Y. Sun, and T. Leiknes, "Monitoring influent measurements at water resource recovery facility using data-driven soft sensor approach," IEEE Sensors Journal, vol. 19, no. 1, pp. 342-352, 2018.

[17] Z. Li, Z. Zou, and L. Wang, "Analysis and forecasting of the energy consumption in wastewater treatment plant," Mathematical Problems in Engineering, vol. 2019, 2019.

[18] D. Torregrossa, U. Leopold, F. Hernández-Sancho, and J. Hansen, "Machine learning for energy cost modelling in wastewater treatment plants," Journal of environmental management, vol. 223, pp. 1061-1067, 2018.

[19] K. B. Newhart, R. W. Holloway, A. S. Hering, and T. Y. Cath, "Data-driven performance analyses of wastewater treatment plants: A review," Water research, 2019.

[20] A. Dairi, T. Cheng, F. Harrou, Y. Sun, and T. Leiknes, "Deep learning approach for sustainable wwtp operation: A case study on data-driven influent conditions monitoring," Sustainable Cities and Society, vol. 50, p. 101670, 2019.

[21] L. Yao and Z. Ge, "Deep learning of semisupervised process data with hierarchical extreme learning machine and soft sensor application," IEEE Transactions on Industrial Electronics, vol. 65, no. 2, pp. 1490-1498, 2017.

[22] H. Darvishi, D. Ciuonzo, E. R. Eide, and P. S. Rossi, "A data-driven architecture for sensor validation based on neural networks," IEEE Sensors conference, 2020.

[23] Z. Pala and R. Atici, "Forecasting sunspot time series using deep learning methods," Solar Physics, vol. 294, no. 5, p. 50, 2019.

[24] J. Ma, Y. Ding, J. C. Cheng, F. Jiang, and Z. Xu, "Soft detection of 5-day bod with sparse matrix in city harbor water using deep learning techniques," Water Research, vol. 170, p. 115350, 2020.

[25] G. Wilkinson and C. Rogers, "Symbolic description of factorial models for analysis of variance," Journal of the Royal Statistical
Society: Series C (Applied Statistics), vol. 22, no. 3, pp. 392-399, 1973.

[26] F. Kadri, F. Harrou, S. Chaabane, Y. Sun, and C. Tahon, "Seasonal arma-based spc charts for anomaly detection: Application to emergency department systems," Neurocomputing, vol. 173, pp. 2102-2114, 2016.

[27] A. C. Harvey, Forecasting, structural time series models and the Kalman filter. Cambridge university press, 1990.

[28] N. Feroze, "Forecasting the patterns of covid-19 and causal impacts of lockdown in top ten affected countries using bayesian structural time series models," Chaos, Solitons \& Fractals, p. 110196, 2020.

[29] M. A. K. Jaradat and M. F. Abdel-Hafez, "Non-linear autoregressive delay-dependent ins/gps navigation system using neural networks," IEEE sensors journal, vol. 17, no. 4, pp. 1105-1115, 2016.

[30] R. J. Hyndman, Y. Khandakar et al., Automatic time series for forecasting: the forecast package for R. Monash University, Department of Econometrics and Business Statistics ..., 2007, no. $6 / 07$.

[31] S. Hochreiter and J. Schmidhuber, "Long short-term memory," Neural computation, vol. 9, no. 8, pp. 1735-1780, 1997.

[32] A. T. Eseye, J. Zhang, and D. Zheng, "Short-term photovoltaic solar power forecasting using a hybrid wavelet-pso-svm model based on scada and meteorological information," Renewable energy, vol. 118, pp. 357-367, 2018

[33] K. Wang, X. Qi, and H. Liu, "A comparison of day-ahead photovoltaic power forecasting models based on deep learning neural network," Applied Energy, vol. 251, p. 113315, 2019.

[34] H. Wang, H. Yi, J. Peng, G. Wang, Y. Liu, H. Jiang, and W. Liu, "Deterministic and probabilistic forecasting of photovoltaic power based on deep convolutional neural network," Energy conversion and management, vol. 153, pp. 409-422, 2017.

[35] K. Cho, B. Van Merriënboer, C. Gulcehre, D. Bahdanau, F. Bougares, H. Schwenk, and Y. Bengio, "Learning phrase representations using rnn encoder-decoder for statistical machine translation," arXiv preprint arXiv:1406.1078, 2014.

[36] J. Honaker, G. King, M. Blackwell et al., "Amelia ii: A program for missing data," Journal of statistical software, vol. 45, no. 7, pp. $1-47,2011$.

[37] F. Harrou, F. Kadri, and Y. Sun, "Forecasting of photovoltaic solar power production using lstm approach," in Advanced Statistical Modeling, Forecasting, and Fault Detection in Renewable Energy Systems. IntechOpen, 2020.

[38] G. Aceto, D. Ciuonzo, A. Montieri, and A. Pescapé, "Toward effective mobile encrypted traffic classification through deep learning," Neurocomputing, vol. 409, no. October, pp. 306-315, 2020 .

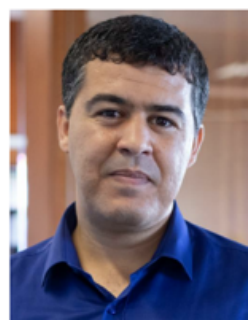

Fouzi Harrou received the M.Sc. degree in telecommunications and networking from the University of Paris VI, France, and the Ph.D. degree in systems optimization and security from the University of Technology of Troyes (UTT), France. He was an Assistant Professor with UTT for one year and with the Institute of Automotive and Transport Engineering, Nevers, France, for one year. He was also a Postdoctoral Research Associate with the Systems Modeling and Dependability Laboratory, UTT, for one year. He was a Research Scientist with the Chemical Engineering Department, Texas A\&M University at Qatar, Doha, Qatar, for three years. He is actually a Research Scientist with the Division of Computer, Electrical and Mathematical Sciences and Engineering, King Abdullah University of Science and Technology. He is the author of more than 130 refereed journals and conference publications and book chapters. He is co-author of the book "Statistical Process Monitoring Using Advanced Data-Driven and Deep Learning Approaches: Theory and Practical Applications" (Elsevier, 2020). His current research interests include statistical decision theory and its applications, fault detection and diagnosis, and deep learning. 


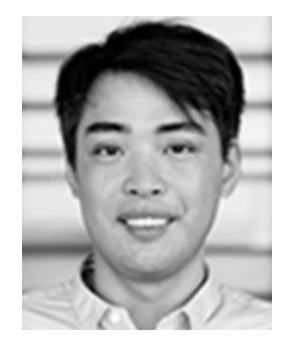

Tuoyuan Cheng received the bachelor's degree in water and wastewater engineering from the Harbin Institute of Technology. He is currently pursuing the $\mathrm{Ph} . \mathrm{D}$. degree in environmental science and engineering with the King Abdullah University of Science and Technology. His current research interests include the application of anomaly detection, data-driven prediction and optimization in water/wastewater treatment plant, and water distribution systems.

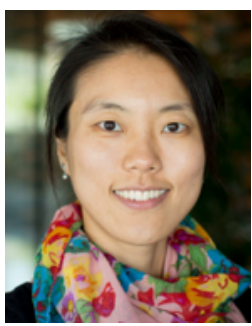

Ying Sun received the Ph.D. degree in statistics from Texas A\&M, in 2011. She held a two-year postdoctoral research position at the Statistical and Applied Mathematical Sciences Institute and the University of Chicago. She was an Assistant Professor with Ohio State University for a year before joining KAUST, in 2014. At KAUST, she established and leads the Environmental Statistics research group, which works on developing statistical models and methods for complex data to address important environmental problems. She has made original contributions to environmental statistics, in particular in the areas of spatiotemporal statistics, functional data analysis, visualization, computational statistics, with an exceptionally broad array of applications. She received two prestigious awards: The Early Investigator Award in Environmental Statistics presented by the American Statistical Association and the Abdel El-Shaarawi Young Research Award from The International Environmetrics Society.

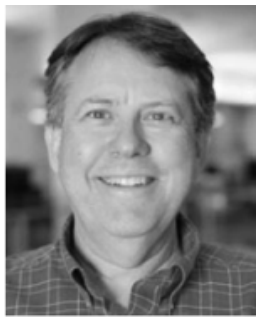

TorOve Leiknes was a Professor with the Department of Hydraulic and Environmental Engineering, Faculty of Engineering Science and Technology, Norwegian University of Science and Technology (NTNU), Trondheim, Norway. In 2013, he joined the King Abdullah University of Science and Technology (KAUST), Saudi Arabia. At NTNU, he was involved in various European Union (EU) projects and a Coordinator of the EUROMBRA Project targeting the development of membrane bioreactor technology for municipal wastewater treatment. At KAUST, his main research activities are focused on the development of novel membrane technologies for the low-energy treatment of waters with impaired water quality for various reuse purposes, using wastewater as a resource for high-value product formation, new concepts of water treatment in RAS, and membrane filtration of PW for alternative reuse strategies. Developing integrated technologies for water reclamation and use in urban agriculture is a new research area of interest. A key component is fundamental studies of biofouling in membrane systems by applying in situ non-destructive techniques. He has more than 20 years' working experience in these areas.

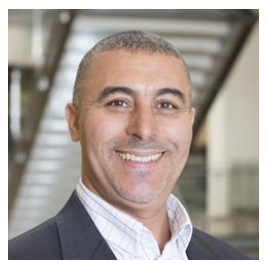

Noreddine Ghaffour received his MSc and $\mathrm{PhD}$ degrees in Energy \& Process Engineering from Montpellier University, France, in 1992 and 1995, respectively. He is currently a Professor of Environmental Science and Engineering at KAUST where he joined in 2010. He has over 27 years of experience in the field of drinking water treatment technologies, and has specialized in the area of membrane and thermal desalination processes. Over the years, he has made major contributions becoming an internationally recognized expert in desalination technologies and its related fields. He received several grants and awards, with the latest Brains for Brine Challenge Award, 2019, the Netherlands. He is the author of over 400 journal and conference publications, several patents and chapters in textbooks. He is a frequent keynote speaker in international conferences, seminars and workshops. He also has experience in scaling-up innovative desalination processes and startups. 